\title{
PHOTOIONIZATION OF HYDROGEN IN ATMOSPHERES OF MAGNETIC NEUTRON STARS
}

\author{
Alexander Y. Potekhin \\ Ioffe Physical-Technical Institute, 194021, St.-Petersburg, Russia; palex@astro.ioffe.rssi.ru \\ and \\ George G. Pavlov \\ Pennsylvania State University, 525 Davey Lab, University Park, PA 16802, U.S.A.; pavlov@astro.psu.edu
}

\begin{abstract}
The strong magnetic fields $\left(B \sim 10^{12}-10^{13} \mathrm{G}\right.$ ) characteristic of neutron stars make all the properties of an atom strongly dependent on the transverse component $\boldsymbol{K}_{\perp}$ of its generalized momentum. In particular, the photoionization process is modified substantially: (i) threshold energies are decreased as compared with those for an atom at rest, (ii) cross section values are changed significantly, and (iii) selection rules valid for atoms at rest are violated by the motion so that new photoionization channels become allowed. To calculate the photoionization cross sections, we, for the first time, employ exact numerical treatment of both initial and final atomic states. This enables us to take into account the quasi-bound (autoionizing) atomic states as well as coupling of different ionization channels. We extend the previous consideration, restricted to the so-called centered states corresponding to relatively small values of $K_{\perp}$, to arbitrary states of atomic motion. We fold the cross sections with the thermal distribution of atoms over $\boldsymbol{K}$. For typical temperatures of neutron star atmospheres, the averaged cross sections differ substantially from those of atoms at rest. In particular, the photoionization edges are strongly broadened by the thermal motion of atoms; this "magnetic broadening" exceeds the usual Doppler broadening by orders of magnitude. The decentered states of the atoms give rise to the low-energy component of the photoionization cross section. This new component grows significantly with increasing temperature above $10^{5.5} \mathrm{~K}$ and decreasing density below $1 \mathrm{~g} \mathrm{~cm}^{-3}$, i. e., for the conditions expected in atmospheres of middle-aged neutron stars.
\end{abstract}

Subject headings: atomic processes: photoionization, autoionization - magnetic fields - stars: neutron

Accepted for publication in The Astrophysical Journal 


\section{INTRODUCTION}

The thermal-like surface radiation detected recently from a number of isolated neutron stars (NSs) (see, e. g., Ögelman 1995 for a review) provides valuable information about the NS temperatures and radii. This enables one to trace the thermal history of NSs and to put constraints on the properties of the superdense matter in NS interiors (e. g., Pethick 1992). However, the observed spectra are substantially modified by reprocessing of the thermal radiation in NS atmospheres (Romani 1987). Pavlov et al. (1995; and references therein) have shown that the strong magnetic fields of NSs, $B \sim 10^{12}-10^{13} \mathrm{G}$, affect the emergent spectra significantly. In particular, photoabsorption by the strongly magnetized atomic hydrogen gas which presumably covers the NS surface should be taken into account. The magnetic field strongly affects the structure and radiative transitions of the hydrogen atom when the electron cyclotron energy $\hbar \omega_{c}=\hbar e B /\left(m_{\mathrm{e}} c\right)$ is much greater than the Rydberg energy Ry $=m_{\mathrm{e}} e^{4} /\left(2 \hbar^{2}\right)=13.6 \mathrm{eV}$, i. e., $\gamma=\hbar \omega_{c} /(2 \mathrm{Ry})=B /\left(2.35 \times 10^{9} \mathrm{G}\right) \gg 1$. In particular, the atom becomes stretched along the magnetic field, the ionization potential is increased by a factor of $\sim \ln ^{2} \gamma$, and radiative transitions become strongly dependent on the photon polarization.

Photoionization of strongly magnetized hydrogen atoms at rest has been considered by a number of authors. The most thorough consideration in the adiabatic approximation, applicable for very large $\gamma$ and $\omega \ll \omega_{c}$ ( $\omega$ is the photon frequency), was presented by Potekhin \& Pavlov (1993; hereafter PP93). This approximation neglects coupling of states with different values of the Landau quantum number $n$ which specifies the energy of the electron motion transverse to the magnetic field (the other quantum number associated with the transverse motion, the projection $s$ of the angular momentum onto the field, is exact for atoms at rest). Potekhin, Pavlov, \& Ventura (1996; hereafter PPV97) went beyond the adiabatic approximation and resolved, in particular, a longstanding contradiction: some authors (e. g., Schmitt et al. 1981; Wunner et al. 1983; Ventura et al. 1992) concluded that the photoionization cross section is exactly zero for photons polarized transversely to the magnetic field, whereas other authors (e. g., Gnedin, Pavlov, \& Tsygan 1973; Miller \& Neuhauser 1991; PP93) obtained finite cross sections. PP93 showed that the discrepancy rooted in different forms of the interaction potential (so-called "velocity form" and "length form") employed by the two groups of authors, and PPV97 proved that the velocity form used by the former group resulted in missing the main contribution to the cross section. The nonadiabatic approach enabled PPV97 to study correlation of different ionization channels and manifestation of autoionization (Beutler-Fano) resonances in the photoabsorption spectra.

The above-cited papers did not take into account the atomic motion inevitable in any real situation. If there were no strong magnetic field, the motion would only lead to the trivial Doppler broadening of the spectral lines and photoionization edges. On the contrary, the finiteness of the atomic velocities in strong magnetic fields leads to many qualitatively new effects (Pavlov \& Mészáros 1993; hereafter PM93) associated with strong distortion of the atomic structure due the Lorentz electric field generated by the motion. Since the moving atom loses its cylindrical symmetry, the projection $s$ of the angular momentum is no longer a good quantum number (in other words, the $s$ adiabatic approach which neglects coupling of states with different $s$ becomes invalid). Hence, the selection rules for the radiative transitions are changed so that new types of transitions become allowed. Since the atomic structure depends on the velocity of the atom, and different atomic states are affected by the motion differently, a new type of broadening of spectral features, magnetic broadening, becomes the most important.

The energies and wave functions of the moving atom depend on the transverse component $K_{\perp}$ of a generalized momentum $\boldsymbol{K}$ (see below), which, contrary to the canonical and kinetic momenta, is conserved for a neutral atom moving in a magnetic field. PM93 analyzed various effects of the motion of the hydrogen atom in the perturbative regime, when $K_{\perp}$ is small. When $K_{\perp}$ exceeds some critical value, the magnetic and (motion-generated) electric fields form a potential well at a distance $r_{c}=m_{\mathrm{e}} K_{\perp} / \omega_{c}$ from the proton, which gives rise to so-called decentered (or shifted) states (Burkova et al. 1976). The decentered states recover the cylindrical symmetry ( $s$ becomes a good quantum number again at large $K_{\perp}$ ), but the axis of symmetry, with respect to which $s$ is defined, is shifted by $r_{c}$. In other words, these states can be described in the shifted $s$-adiabatic approximation. Binding energies, wavefunctions and oscillator strengths of radiative transitions between the 
discrete levels of an arbitrarily moving $\mathrm{H}$ atom (beyond the adiabatic approximation) have been studied by Potekhin (1994; hereafter P94). Our recent study of the hydrogen bound-bound opacity in NS atmospheres (Pavlov \& Potekhin 1995; hereafter PP95) was based on these results.

Less investigated are effects of the motion across the field on the bound-free radiative transitions of atoms. PM93 have shown, in the perturbation approach, that the motion strongly affects photoionization cross sections through opening and enhancement of additional photoionization channels forbidden for the non-moving atom due to its cylindrical symmetry, and through the strong magnetic broadening of the photoionization edges. Bezchastnov \& Potekhin (1994; hereafter BP94) suggested a modification of the adiabatic approach which allows for different displacements of the initial and final states and presented a convenient formalism for calculating the transition rates between such states. They have also performed calculations with the initial state treated non-adiabatically; the two approaches exhibited a fair agreement with each other. Kopidakis, Ventura \& Herold (1996; hereafter KVH96) also went beyond the perturbation approximation and calculated the frequency dependences of the cross sections for various values of $K_{\perp}$, assuming these values small enough to neglect the decentered bound states. The results of the two latter papers differ from each other by orders of magnitude for radiation polarized transversely to the field. The origin of the discrepancy is the same as for atoms at rest - KVH96 employed the velocity form of the interaction potential which led to wrong results when used with the $n$-adiabatic approximation which treats the Landau number $n$ as the exact quantum number. Both BP94 and KVH96 treated final (continuum) states of electron in the (shifted) adiabatic approximation, neglecting coupling of final states with different $s$ and contribution of quasibound (autoionizing) states to the photoionization cross section.

The general non-adiabatic numerical approach developed by PPV97 for the continuum wave functions of the strongly magnetized hydrogen has been applied there to the particular case of non-moving atoms. In the present work, we employ that approach, together with the theoretical formalism of P94 and BP94, for an accurate treatment of both discrete and continuum states of the moving atom and the radiative transitions between them. Unlike BP94 and KVH96, we do not restrict ourselves to the case of relatively small generalized momenta of atoms, but consider also the decentered states which arise at large $K_{\perp}$. This enables us to calculate the cross sections of photoionization by arbitrarily polarized photons of the hydrogen atom arbitrarily moving in a magnetic field $B \sim 10^{12}-10^{13} \mathrm{G}$. Averaging these cross sections with thermal distributions of atoms yields the boundfree opacities needed for modeling NS atmospheres.

In Section 2 we outline basic techniques for numerical treatment of a strongly magnetized $\mathrm{H}$ atom and its interactions with radiation (the general form of the interaction matrix elements is given in the Appendix). In Section 3 we present the photoionization cross sections of $\mathrm{H}$ atoms in various states of motion across the magnetic field and cross sections averaged over thermal motion for conditions typical for atmospheres of isolated NSs.

\section{THEORETICAL FRAMEWORK}

\subsection{Generalized momentum and decentering of $\mathbf{H}$ atoms}

The center-of-mass motion of an atom in a magnetic field can be conveniently described by the generalized momentum $\boldsymbol{K}$ (Gorkov \& Dzyaloshinskii 1968; see also Johnson, Hirschfelder, \& Yang 1983),

$$
\boldsymbol{K}=\boldsymbol{P}-\frac{e}{2 c} \boldsymbol{B} \times\left(\boldsymbol{r}_{e}-\boldsymbol{r}_{\mathrm{p}}\right),
$$

where $\boldsymbol{P}$ is the canonical momentum, $\boldsymbol{r}_{\mathrm{e}}-\boldsymbol{r}_{\mathrm{p}}$ is the relative coordinate, the subscript "e" or "p" indicates electron or proton, respectively, and the cylindric gauge of the vector potential, $\boldsymbol{A}(\boldsymbol{r})=\frac{1}{2} \boldsymbol{B} \times \boldsymbol{r}$, is used. We shall consider the representation in which all three components of $\boldsymbol{K}$ have definite values. Then the two-body wavefunction can be factorized into a phase factor depending on the center-of-mass coordinate and the wavefunction $\psi_{\boldsymbol{K}}$ depending on $\boldsymbol{r}_{\mathrm{e}}-\boldsymbol{r}_{\mathrm{p}}$.

It is useful to define a basic electron-proton separation $\eta \boldsymbol{r}_{c}$ and to regard the deviation from it, $\boldsymbol{r}=\boldsymbol{r}_{\mathrm{e}}-\boldsymbol{r}_{\mathrm{p}}-\eta \boldsymbol{r}_{c}$, as an independent variable. Here $\boldsymbol{r}_{c}=\left(c / e B^{2}\right) \boldsymbol{B} \times \boldsymbol{K}$ is the position of the relative guiding center, and $\eta$ is the shift parameter. It is also convenient to use a special axial gauge of the vector potential (Vincke, Le Dourneuf, \& Baye 1992; P94). Then we arrive at a set of Hamiltonians $H^{(\eta)}$ and wavefunctions $\psi^{(\eta)}(\boldsymbol{r})$ depending on $\eta$. In the particular cases of $\eta=0$ and $\eta=1$, the "conventional" and "shifted" representations of Gorkov \& Dzyaloshinskii 
(1968) are recovered. Wavefunctions with different $\eta$ are related to each other by the phase transformation (BP94)

$$
\psi_{\boldsymbol{K}}^{(\eta)}(\boldsymbol{r})=\exp \left[-\frac{\mathrm{i}}{\hbar} \frac{m_{\mathrm{p}}-m_{\mathrm{e}}}{2 m_{\mathrm{H}}} \eta \boldsymbol{K} \boldsymbol{r}_{\perp}\right] \psi_{\boldsymbol{K}}^{(0)}\left(\boldsymbol{r}+\eta \boldsymbol{r}_{c}\right)
$$

where $\boldsymbol{r}_{\perp}=(x, y)$ is the coordinate transverse to the field $\boldsymbol{B}$, and $m_{\mathrm{H}}=m_{\mathrm{e}}+m_{\mathrm{p}}$ is the total mass of the atom.

If there were no Coulomb attraction, then the electron Landau number $n=0,1,2, \ldots$ and the $z$ projection of the angular momentum of the relative motion $s \geq-n$ would be exact quantum numbers. In this case the energy of the transverse motion (with the constant minimum energy subtracted) is

$$
E_{n s}^{\perp}=\left[n+\left(m_{\mathrm{e}} / m_{\mathrm{p}}\right)(n+s)\right] \hbar \omega_{c}
$$

and the transverse part of the wave function can be described (for a given $\boldsymbol{K}_{\perp}$ ) by a Landau function

$$
\Phi_{n s}\left(\boldsymbol{r}_{\perp}^{\prime}\right)=\frac{1}{a_{\mathrm{m}} \sqrt{2 \pi}} \mathrm{e}^{-\mathrm{i} s \phi} I_{n+s, n}\left(\frac{{r^{\prime}}_{\perp}}{2 a_{\mathrm{m}}^{2}}\right)
$$

which depends on $\boldsymbol{r}_{\perp}^{\prime}=\boldsymbol{r}_{e \perp}-\boldsymbol{r}_{p \perp}-\boldsymbol{r}_{c}\left(=\boldsymbol{r}_{\perp}(\eta=1)\right)$. Here $\phi$ is the azimuthal angle of $\boldsymbol{r}_{\perp}^{\prime}, a_{\mathrm{m}}=(\hbar c / e B)^{1 / 2}$ is the magnetic length, $I_{n n^{\prime}}$ is a Laguerre function (Sokolov \& Ternov 1968), and the $z$-projection $s$ of the angular momentum of the relative motion is defined in the shifted reference frame, $\eta=1$ (since $\boldsymbol{K}_{\perp}$ is definite, the electron and proton do not possess definite $z$-projections of the angular momenta separately from each other - see Johnson et al. 1983). Equation (4) implies that after photoionization of a moving hydrogen atom, when the photoelectron moves away to large distances at which the Coulomb interaction can be neglected, it acquires the displacement $\boldsymbol{r}_{c}$ from the proton in the transverse plane.

\subsection{Atomic wavefunctions}

A wavefunction $\psi_{\kappa}^{(\eta)}$ of an atomic state $|\kappa\rangle$ can be expanded over the complete set of the Landau functions

$$
\psi_{\kappa}^{(\eta)}(\boldsymbol{r})=\sum_{n s} \Phi_{n s}\left(\boldsymbol{r}_{\perp}\right) g_{n, s ; \kappa}^{(\eta)}(z)
$$

(note that $\boldsymbol{r}_{\perp}$ depends on $\eta$ ). The adiabatic approximation would correspond to retaining only one term in this expansion.
A bound state of the atom can be numbered as $|i\rangle=\left|n_{i}, s_{i}, \nu, \boldsymbol{K}\right\rangle$, where $n_{i}$ and $s_{i}$ relate to the leading term of the expansion (5), and $\nu$ enumerates longitudinal energy levels

$$
E_{i}^{\|}=E_{i}-E_{n_{i} s_{i}}^{\perp}
$$

and controls the $z$-parity: $g_{n, s ; \kappa}^{(\eta)}(-z)=(-1)^{\nu} g_{n, s ; \kappa}^{(\eta)}(z)$. For a moving atom, this way of numbering is unambiguous at $\gamma \gtrsim 300$ (P94).

Since the transverse factors $\Phi_{n s}$ in equation (5) are known analytically, only the one-dimensional longitudinal functions $g_{n s ; \kappa}^{(\eta)}$ are to be found numerically. For the bound states, numerical calculations (Vincke et al. 1992; P94) yield the following. At small transverse generalized momenta $K_{\perp}$, the states $\nu=0$ remain tightly bound and centered, with the electron cloud concentrated around the proton. For such states, the conventional representation $(\eta=0)$ is appropriate. At large $K_{\perp}$, the states are decentered, with the electron localized in a potential well shifted apart from the proton. Then $\eta=1$ is the apt choice. With growing $K_{\perp}$, the transition from the centered tightly-bound states to the decentered states occurs in a narrow range of $K_{\perp}$ near $K_{\mathrm{c}} \simeq \sqrt{2 m_{\mathrm{H}}\left|E^{\|}\right|}$, and the shifted $(\eta=1)$ adiabatic approximation becomes fairly good at $K_{\perp} \gg K_{\mathrm{c}}$. For the hydrogen-like states $\nu \geq 1$, however, the mean electron-proton separation grows steadily, being close to $r_{c}$ at arbitrarily small $K_{\perp}$, so that these states can be described by the shifted adiabatic approximattion at any $K_{\perp}$. Finally, at very large $K_{\perp}\left(\gg \gamma \hbar / a_{\mathrm{B}}\right.$, where $a_{\mathrm{B}}$ is the Bohr radius) the longitudinal functions become oscillatorlike for any states, corresponding to a wide, shallow parabolic potential well of a depth $\simeq e^{2} / r_{c}$ below the continuum boundary (Burkova et al. 1976; P94).

A continuum state can be numbered as $|f\rangle=$ $\left|n_{f}, s_{f}, E_{f}, I, \boldsymbol{K}^{\prime}\right\rangle$, where $n_{f}$ and $s_{f}$ correspond to an open channel for the energy $E_{f}$, and $I= \pm 1$ corresponds to a type of the final state - e. g., to a given $z$-parity or to a direction of electron escape, depending on asymptotic conditions. In the photoionization process the value of $\boldsymbol{K}^{\prime}$ is constrained by the momentum conservation law: $\boldsymbol{K}^{\prime}=\boldsymbol{K}+\hbar \boldsymbol{q}$, where $\boldsymbol{q}$ is a photon wavevector.

For the continuum without motion $\left(K_{\perp}^{\prime}=0\right)$, a technique for calculating the longitudinal wavefunctions has been developed by PPV97. It can be easily extended to the general case, $K^{\prime} \neq 0$, by numerating the orbitals $n s$ in equation (5) for the state $|f\rangle$ 
with single integers $j$ such that larger values of $j$ correspond to higher energies $E_{n s}^{\perp}$. Since the Coulomb interaction ceases to distort the transverse wavefunctions at infinity, the orbitals in equation (5) become uncoupled at large $z$ provided that the full-shift representation $\left(\eta^{\prime}=1\right)$ of the continuum state is chosen. We shall adopt this choice hereafter.

\subsection{Interaction with radiation}

The cross section for ionizing an atomic state $|i\rangle$ into a continuum state $|f\rangle$ by a photon with frequency $\omega$, wavevector $\boldsymbol{q}$, and polarization vector $\boldsymbol{e}$ is

$$
\sigma_{i \rightarrow f}=\pi \frac{e^{2}}{\hbar c} \frac{\mathrm{Ry}}{\hbar \omega}\left(\frac{\mathrm{Ry}}{E_{f}^{\|}} \frac{\mu}{m_{\mathrm{e}}}\right)^{1 / 2} L_{z} a_{\mathrm{B}}|\langle f|\hat{M}| i\rangle|^{2},
$$

where $\mu$ is the reduced mass, $L_{z}$ is the $z$-extension of the periodicity volume of the final state, and $\hat{M}$ is the dimensionless interaction operator. For an arbitrarily moving atom of finite mass, the expression for $\hat{M}$ is given in the Appendix. Using equation (5) for both the initial and final atomic states, we obtain

$$
\langle f|\hat{M}| i\rangle=\sum_{n s n^{\prime} s^{\prime}}\left\langle n^{\prime} s^{\prime} \eta^{\prime}, f\left|\left\langle n^{\prime} s^{\prime} \eta^{\prime}|\hat{M}| n s \eta\right\rangle_{\perp}\right| n s \eta, i\right\rangle_{\|},
$$

where $|n s \eta, \kappa\rangle_{\|}$corresponds to the longitudinal wavefunction $g_{n s ; \kappa}^{(\eta)}(z)$, and $|n s \eta\rangle_{\perp}$ corresponds to $\Phi_{n s}$. The interaction operator $\hat{M}$ in this equation depends on $\eta^{\prime}, \eta$, because the common phase factor (eq. [2]) has been included in it (see the Appendix).

The Landau functions for the initial and final states in equation (8) depend on differently displaced arguments if $\eta^{\prime} \neq \eta$. This is the case for the tightly bound initial states at $K_{\perp}<K_{\mathrm{c}}$, for which the apt choice of the shift parameter is $\eta=0$ (while $\left.\eta^{\prime}=1\right)$. The different displacements strongly complicate explicit integration in the inner (transverse) matrix elements.

In order to reduce the initial and final wavefunctions in the same basis, KVH96 expressed the displaced Landau state as a series over the undisplaced states. This reexpansion involves an additional summation index which runs over a huge number of values if the relative displacement is large enough, which renders the reexpansion method impractical at high $K_{\perp}$.

The difficulty can be circumvented using the creationannihilation operator formalism of BP94. Analytical calculation of $\left\langle n^{\prime} s^{\prime} \eta^{\prime}|\hat{M}| n s \eta\right\rangle_{\perp}$ is performed in the
Appendix. As a result, the interaction matrix element $\langle f|\hat{M}| i\rangle$ reduces to a sum of one-dimensional quadratures (eq. A9]) feasible for numerical evaluation.

\section{RESULTS AND DISCUSSION}

\subsection{Photoionization at fixed $\mathrm{K}_{\perp}$}

Many properties of the photoionization cross section can be understood from the $K_{\perp}$-dependence of the level energies shown in Figure 1. The monotonic increase of the energies shifts the photoionization thresholds redward. Admixture of many orbitals near "anticrossings" of levels belonging to different $s$-manifolds (e. g., at $K_{\perp} \approx 150$ a.u.) causes peculiarities in the radiative transition rates (P94). The levels which lie above zero in Figure 1 correspond to quasi-bound states in the continuum. They give rise to sharp resonances in the cross sections at the photon energies corresponding to transitions between the initial bound state and the quasi-bound states.

Figure 2 demonstrates a comparison of our numerical results (solid lines) with those obtained using approximations encountered in literature. The short-dash-dot curves are obtained using the velocity form of the interaction operator with the $s^{\prime}$-coupling "switched-off" ( $s^{\prime}$-adiabatic approximation). We see that this coupling is very important for evaluating the cross section $\sigma_{-}$(for the left circular polarization transverse to the magnetic field) and less important for the other two basic cross sections, $\sigma_{+}$(right circular polarization transverse to the field) and $\sigma_{\|}$(linear polarization along the field). The dotted lines are obtained using the shifted adiabatic approximation for the final state and the length representation of the interaction operator. Note that they are close to the short-dash-dot lines in the upper panels. This is in accordance with approximate equivalence of using the $n$-adiabatic approximation $\left(n, n^{\prime}=0\right)$ with the length form and using the velocity form with allowance for $n, n^{\prime}>0$. This equivalence has been suggested by PP93 and proved by PPV97 under the condition that the non-adiabatic corrections were small. It fails for $K_{\perp} \sim K_{\mathrm{c}}$ if the $s^{\prime}$-coupling is neglected (compare dotted and dot-short-dash curves in the lower panels), but we have checked numerically that it holds if the $s, s^{\prime}$-coupling is included.

The dot-long-dash curves correspond to the approach of BP94. It differs from the plain shifted $\left(n^{\prime} s^{\prime}\right)$-adiabatic approximation (dotted curves) by employing the orthogonalization of the final state with 
respect to the initial one (see eq. [32] of BP94). At relatively small $K_{\perp}$ (upper panels), this approximation yields qualitatively correct results. With increasing $K_{\perp}$, the approximate $\sigma_{+}$becomes progressively less accurate due to violation of the cylindrical symmetry of the continuum states of the atom; for instance, it exceeds our cross section by a factor of $\sim 5-7$ at $K_{\perp}=100$ a.u. However, the approximate $\sigma_{-}$and $\sigma_{\|}$ remain surprisingly accurate.

Short dashes correspond to the approach of KVH96, who use the $n$-adiabatic approximation for the initial state, and the shifted adiabatic and Born approximations for the final state. If to abandon the Born approximation (retaining the adiabatic one), then $\sigma_{ \pm}$ would become considerably lower, by 0.5 to 3 orders of magnitude for the parameters chosen in Fig. 2, and even more different from the true cross sections. This lowering is due to the approximate orthogonality of the initial and final longitudinal wave functions, which is not provided by the Born approximation.

All the discussed approximations miss the BeutlerFano resonances (narrow peaks and dips near the thresholds), which occur at the energies of the quasibound states in the continuum. These resonances are analogous to those discussed by PPV97, except that now these quasi-bound states all belong to the $\left(n^{\prime}=0\right)$-manifold. Autoionization in this case becomes possible due to the motion-induced coupling of different $s^{\prime}$-channels.

Except for the missing resonances, the discussed approximations appear to be sufficiently accurate for the longitudinal $\left(\sigma_{\|}\right)$polarization.

Figure 3 demonstrates a representative sample of our numerical results for $\gamma=1000$. In accordance with Figure 1, the photoionization thresholds shift redward with increasing $K_{\perp}$. Since $K_{\mathrm{c}} \approx 150$ a.u. at this field strength, the left and right panels correspond to the centered and decentered atoms, respectively.

For the centered atoms, the slopes of the curves for each given polarization remain practically coincident with those at $K_{\perp}=0$. The values of the cross sections for the parallel and right polarizations, corresponding to the radiative transitions with $s^{\prime}=s$ and $s^{\prime}=s+1$ allowed at $K_{\perp}=0$ (when $s$ is a "good" quantum number), decrease only slightly with increasing $K_{\perp}$ from 0 to $K_{\mathrm{c}}$. For the left polarization, radiative transitions from the ground state are forbidden $\left(\sigma_{-}=0\right)$ at $K_{\perp}=0$ (PP93). With growing $K_{\perp}$, the coupling between different $s$-channels arises, and $\sigma_{-}$grows (approximately as $K_{\perp}^{2}-$ see PM93) until it becomes comparable to $\sigma_{+}$at $K_{\perp} \sim K_{\mathrm{c}}$.

For the decentered atoms (right panels of Fig. 3), the energy dependences steepen with growing $K_{\perp}$. This is explained by the change of the effective potential from Coulomb-like at $K_{\perp} \lesssim K_{\mathrm{c}}$ to oscillator-like as the atom becomes decentered. The effective potentials corresponding to different $s$ become nearly identical, that results in a significant decrease of $\sigma_{+}$. Still faster is the decrease of $\sigma_{-}$caused by restoring applicability of the $s$-adiabatic approximation and corresponding selection rules at large $K_{\perp}$. At the same time, the threshold value of $\sigma_{\|}$remains almost independent of $K_{\perp}$.

Since the moving atom loses its cylindrical symmetry, the cross sections depend on the angle $\varphi$ between $\boldsymbol{K}_{\perp}$ and the transverse component of the wavevector. The $\varphi$-dependence is most pronounced when both the wavevector $\boldsymbol{q}$ and polarization direction $\boldsymbol{e}$ are perpendicular to $\boldsymbol{B}$. The cross sections $\sigma_{\|}$and $\sigma_{\perp}$ are drawn in Figure 3 by dot-long-dash and dashed lines, respectively. The former cross section does not depend on $\varphi$ in the dipole approximation, whereas the small non-dipole corrections (eq. A10d) cause a weak dependence with an amplitude $\ll 1 \%$. Therefore, we show only one curve for $\sigma_{\|}$on each panel. For the transverse polarization, the $\varphi$-dependence becomes pronounced at $K_{\perp} \gtrsim \hbar / a_{\mathrm{m}} \approx 30$ a.u. The upper and lower dashed curves correspond to the largest and smallest possible values of $\sigma_{\perp}(\varphi)$, at $\varphi=0$ and $\varphi=90^{\circ}$, respectively. The reason is that the transversely polarized photons "see" the largest transverse size of the atom (stretched perpendicular to $\boldsymbol{K}_{\perp}$ ) when they propagate along $\boldsymbol{K}_{\perp}$, and the smallest one when propagating transversely to $\boldsymbol{K}_{\perp}$. The $\varphi$ dependence between these two extremes, illustrated in Figure 1 , is described by the formula

$$
\sigma_{\perp}(\varphi)=\frac{\sigma_{+}+\sigma_{-}}{2}+A \cos 2 \varphi .
$$

Note that in the absence of coupling of different $s$ channels the amplitude $A$ in equation (9) would equal $\sqrt{\sigma_{+} \sigma_{-}}$, which is always greater than the actual value of $A$, calculated numerically.

The curve corresponding to $E_{f}=1.1$ Ry in Figure 1 goes higher than that corresponding to $E_{f}=1$ Ry because the ionization channel $s^{\prime}=1$ opens at $E_{f}=\hbar \omega_{c \mathrm{p}}=1.089 \mathrm{Ry}$, where $\omega_{c \mathrm{p}}=\left(m_{\mathrm{e}} / m_{\mathrm{p}}\right) \omega_{c}$ is the proton cyclotron frequency. The curve corre- 
sponding to $E_{f}=2$ Ry goes much higher than the other curves because it falls onto a slope of a BeutlerFano resonance. Such resonances are most prominent at $K_{\perp} \sim K_{\mathrm{c}}$ (cf. Fig. 3) because of the violation of the $s$-adiabatic approximation in this range of the transverse generalized momenta. Nevertheless, these peaks remain very narrow. Figure 5 presents some autoionization widths $\Gamma_{\mathrm{a}}$ calculated according to equations (14) and (15) of PPV97 (with obvious generalization to the case of moving atoms). The left panel shows the widths of the tightly-bound states $s=1$ through 4 which enter the continuum at $K_{\perp} \approx 1200,560,340$, and 237 a.u., respectively (see Fig. 11). Owing to the similarity of the effective potentials related to different $s$ values, the jumps of the curves corresponding to opening of additional autoionization channels occur at nearly the same values of $K_{\perp}$. The hydrogen-like levels of the $(s=1)$-manifold (right panel of Fig. 5) belong to the continuum at any $K_{\perp}$. The autoionization widths vanish at $K_{\perp} \ll K_{\mathrm{c}}$ and at $K_{\perp} \gg K_{\mathrm{c}}$ because of restoring cylindrical symmetry of the wavefunctions.

\subsection{Absorption by atoms in thermal equilib- rium}

The bound-free absorption coefficient for a hydrogen gas of given temperature and density is proportional to the photoionization cross section $\sigma_{\kappa \rightarrow \kappa^{\prime}}\left(\boldsymbol{K}_{\perp}\right)$ folded with $N_{\kappa}(\boldsymbol{K})$, the distribution of the number density of atoms in the initial state. Since the level energy $E_{\kappa}\left(K_{\perp}\right)$ tends to a constant value at $K_{\perp} \rightarrow \infty$, the normalization integral for the Boltzmann distribution $N_{\kappa}(\boldsymbol{K}) \propto \exp \left[-E_{\kappa}\left(K_{\perp}\right) / k_{\mathrm{B}} T\right]$ diverges. The divergence is eliminated if nonideality of the gas is taken into account. At high $K_{\perp}$, the binding energies become small, and atomic sizes become large. This means that the atom can be easily destroyed by surrounding particles, so that very high $K_{\perp}$ should not contribute to the corresponding integrals. In order to allow for this effect, we use the occupation probability formalism, described in detail by Hummer \& Mihalas (1988) and generalized to the case of the $K_{\perp-}$ dependent atomic structure by PP95. Following this approach, we write

$$
N_{\kappa}(\boldsymbol{K}) \propto w_{\kappa}\left(K_{\perp}\right) \exp \left[-E_{\kappa}\left(K_{\perp}\right) / k_{\mathrm{B}} T\right],
$$

where $w_{\kappa}\left(K_{\perp}\right)$ is the occupation probability, equal to the fraction of atoms (in the specified state $|\kappa, \boldsymbol{K}\rangle$ ) which essentially preserve quantum-mechanical (particularly, optical) properties of an isolated atom. We estimate $w_{\kappa}\left(K_{\perp}\right)$ according to equation (14) of PP95, based on the quantum-mechanical atomic sizes calculated by $\mathrm{P} 94$. A rapid decrease of $w_{\kappa}\left(K_{\perp}\right)$ at $K_{\perp} \gtrsim \gamma \hbar / a_{\mathrm{B}}$ (see Fig. 3 of PP95) limits contribution from atoms with large generalized momenta and provides convergence of integrals over $K_{\perp}$.

Since the threshold energies decrease with increasing $K_{\perp}$, folding the photoionization cross sections with $N_{\kappa}\left(K_{\perp}\right)$ leads to the redward broadening of the photoionization edges, with a typical width $\Gamma_{M} \sim$ $k_{B} T$ (PM93). It has been shown by PM93 and PP95 that the "magnetic width" is much greater than the Doppler and (impact) collisional widths under typical conditions of NS atmospheres. In this paper we take full account of the magnetic broadening and neglect other broadening mechanisms.

The averaged photoionization cross sections for atoms on the ground level are shown in Fig. 6 for two values of temperature $T$. For comparison, we show also cross sections for the bound-bound (b-b) transitions from the ground level (PP95). Both the b-b and bound-free (b-f) averaged cross sections are corrected for the stimulated emission, i. e., multiplied by $\left(1-\exp \left[-\hbar \omega / k_{B} T\right]\right)$. For the circular polarizations, the b-b and b-f transitions dominate in different (adjacent) spectral ranges - the low-energy b-f component, arising from the decentered states (cf. Fig. 3), turns out to be much weaker than the b-b component in the same energy range. For $\sigma_{\|}$, this is also the case at $\log T=5.2$, but not at $\log T=5.8$. In the latter case, the low-energy b-f component dominates the absorption spectrum, except at very low energies $\hbar \omega \lesssim 40 \mathrm{eV}$.

The peak of $\sigma_{+}$in the right panel of Figure 6 at $\hbar \omega \approx 15 \mathrm{eV}$ and the corresponding peaks of the 'b-b' curves arise near the proton cyclotron frequency $\omega_{c \mathrm{p}}$, around which the transitions of strongly decentered atoms are concentrated (cf. PP95).

Figure 7 demonstrates the effect of density on the averaged photoionization cross sections. With increasing $\rho$, the low-energy component rapidly decreases because the decentered atoms, due to their large sizes, are easier destroyed by the plasma microfields. The growth of the high-energy component noticeable at higher $T$ is explained by the relative increase of the number of centered atoms when the decentered atoms are destroyed. It should be noted that at high densities one more effect, broadening of the photoionization edges by quasi-static interactions with surrounding particles, may become important. 
As the density grows, highly excited bound states become randomly disturbed by the surrounding and form a quasi-continuum. This results in continuous photon absorption below the undisturbed photoionization threshold (e. g., Däppen, Anderson, \& Mihalas 1987). These "bound-quasi-free" transitions require a separate treatment and are not included in the present paper.

Figure 8 shows the effects of $T$ and $B$ on the photoabsorption spectral shapes. With growing $T$, the low-energy components significantly increase, and the high-energy components of $\sigma_{\|}$and $\sigma_{+}$decrease slightly, because the decentered states become more populated. At lower $T$, the high-energy component of $\sigma_{-}$grows with $T$ linearly,

$$
\sigma_{-} \approx \frac{\hbar \omega_{c \mathrm{p}} k_{B} T}{2 \epsilon_{000,010}^{2}} \sigma_{+},
$$

in accordance with the result of PM93 obtained in the perturbation regime $\left(\epsilon_{000,010}\right.$ is the energy of the main b-b transition for the right polarization - e. g., $\epsilon_{000,010} \simeq 70 \mathrm{eV}$ for $\gamma=1000$ ). At higher $T$, the growth of $\sigma_{-}$decelerates because of decreasing fraction of the centered states and nonperturbative effects.

At higher $B$, the above-discussed proton cyclotron resonance for $\sigma_{+}$becomes stronger. Owing to the approximate similarity of the energy spectra related to different $s$-manifolds (see Fig. 1), the individual Beutler-Fano resonances, although smoothed by the thermal averaging, are collected together into regularly placed peaks. Such peaks corresponding to four proton cyclotron harmonics are indicated by arrows for $\sigma_{-}$at $\gamma=3000$ in Fig. 8. At high densities, the peaks can be further smoothed by interaction with surrounding particles.

\section{CONCLUSIONS}

The results presented here provide, together with the results of PP95 on the bound-bound transitions, a basis for calculations of realistic opacities of NS atmospheres. To find the opacities, one should add transitions from excited atomic levels, which is straightforward. More difficult are the problems of ionization equilibrium in the dense, non-ideal plasmas and closely related problem of the non-ideality effects on the bound-free transitions (including transitions to the quasi-free states below the unperturbed photoionization threshold). Finally, polarizations of the nor- mal modes for any frequency and direction of propagation should be found for solving the radiative transfer equations. Bulik \& Pavlov (1996) have described how these polarizations can be calculated if the basic cross sections $\sigma_{ \pm}$and $\sigma_{\|}$are known.

Our results show that atomic motion in strong magnetic fields drastically changes both the boundbound and bound-free opacities. In particular, it considerably broadens the spectral lines and photoionization edges (with a typical magnetic width $\sim k_{B} T$ ), gives rise to additional absorption at low frequencies due to decentered atoms, and opens new transition channels forbidden for atoms at rest. These unusual properties of the spectral opacities alter the structure of the NS atmospheres and manifest themselves in the spectra and light curves of radiation emergent from the NS surface layers.

The authors are grateful to Victor Bezchastnov and Joseph Ventura for fruitful discussions, and to Cole Miller and the referee, Tomek Bulik, for useful remarks. This work was partially supported through NASA grant NAG5-2807, INTAS grant 943834, RBRF grant 96-02-16870a, and DFG-RBRF grant 96-02-00177G. AYP gratefully acknowledges the hospitality of Joseph Ventura at the University of Crete, Gilles Chabrier at ENS-Lyon, and Chris Pethick at Nordita, where a part of this work has been done. 


\section{APPENDIX: INTERACTION MATRIX ELEMENTS}

Velocity and length representations of the operator of interaction of a moving hydrogen atom with radiation have been derived by BP94. Here we employ the velocity representation as the simpler one. KVH96 have noted that the interaction of the radiation field with the electron spin should be taken into account. It has been found by PPV97 that the spin term almost does not change the cross sections at energies below $10 \mathrm{keV}$. Nevertheless, we include this term for the sake of generality, neglecting the terms responsible for the spin-flip transitions (Schmitt et al. 1981) which are strictly forbidden at the considered photon energies below $\hbar \omega_{c}$.

In the conventional representation of the wavefunctions, equation (A6) of BP94 yields for the dimensionless interaction operator $\hat{M}$ :

$$
\left(2 a_{\mathrm{B}}\right)^{-1} \hat{M}=\exp \left(\mathrm{i} \frac{m_{\mathrm{p}}}{m_{\mathrm{H}}} \boldsymbol{q} \boldsymbol{r}\right)\left[\left(\frac{\boldsymbol{\pi}}{\hbar}+\frac{m_{\mathrm{e}}}{m_{\mathrm{H}}} \frac{\boldsymbol{K}}{\hbar}+\frac{\boldsymbol{q}}{2}\right) \boldsymbol{e}-\frac{\mathrm{i}}{2}(\boldsymbol{q} \times \boldsymbol{e})_{z}\right]+\left(\frac{m_{\mathrm{e}}}{m_{\mathrm{p}}} \frac{\boldsymbol{\Pi}}{\hbar}-\frac{m_{\mathrm{e}}}{m_{\mathrm{H}}} \frac{\boldsymbol{K}}{\hbar}\right) \boldsymbol{e},
$$

where $\boldsymbol{q}$ is the photon wavevector, $\boldsymbol{e}$ is its polarization vector, and negligibly small terms $\sim\left(m_{\mathrm{e}} / m_{\mathrm{p}}\right) q$ are omitted. Here $\boldsymbol{r}=\boldsymbol{r}_{\mathrm{e}}-\boldsymbol{r}_{\mathrm{p}}$ is the relative coordinate, and $\boldsymbol{\pi}$ and $\boldsymbol{\Pi}$ are the electron- and proton-type kinetic momentum operators:

$$
\begin{aligned}
& \boldsymbol{\pi}=\boldsymbol{p}+\frac{e}{2 c} \boldsymbol{B} \times \boldsymbol{r}, \\
& \boldsymbol{\Pi}=\boldsymbol{p}-\frac{e}{2 c} \boldsymbol{B} \times \boldsymbol{r} .
\end{aligned}
$$

Their cyclic components act on the Landau states as

$$
\begin{aligned}
\pi_{ \pm}|n s\rangle_{\perp} & =\mp \frac{\mathrm{i} \hbar}{a_{\mathrm{m}}} \sqrt{n_{*}}|n \pm 1, s \mp 1\rangle_{\perp}, \\
\Pi_{ \pm}|n s\rangle_{\perp} & =\mp \frac{\mathrm{i} \hbar}{a_{\mathrm{m}}} \sqrt{n_{*}+s}|n, s \mp 1\rangle_{\perp},
\end{aligned}
$$

where $n_{*}=n+1$ and $n_{*}=n$ for the right $(+)$ and left $(-)$ components, respectively. If the full-shift representation, $\eta^{\prime}=1$, is used for the final state, and an arbitrary shift parameter $\eta$ for the initial one, then, according to equation (A15) of BP94,

$$
\begin{aligned}
\hat{M} & =\frac{2 \hbar}{e^{2}} \exp \left[-\frac{\mathrm{i}}{2} \frac{m_{\mathrm{p}}-m_{\mathrm{e}}}{m_{\mathrm{H}}}(1-\eta) \boldsymbol{K} \boldsymbol{r}_{\perp}\right] \\
& \times \boldsymbol{e}\left(\exp \left[\mathrm{i}\left(\boldsymbol{q} \boldsymbol{r}_{\perp} / 2+q_{z} z\right)\right] \boldsymbol{F}_{\mathrm{e}}+\exp \left[-\mathrm{i} \eta \boldsymbol{r}_{c} \boldsymbol{q}-\mathrm{i} \boldsymbol{q} \boldsymbol{r}_{\perp} / 2\right] \boldsymbol{F}_{\mathrm{p}}\right)
\end{aligned}
$$

where $\boldsymbol{r}=\boldsymbol{r}_{\mathrm{e}}-\boldsymbol{r}_{\mathrm{p}}-\eta \boldsymbol{r}_{c}$. Once again, the terms $\sim q m_{\mathrm{e}} / m_{\mathrm{p}}$ are omitted. The components of the vector operators $\boldsymbol{F}_{\mathrm{e}}$ and $\boldsymbol{F}_{\mathrm{p}}$ are

$$
\begin{gathered}
F_{\mathrm{e}+}=\frac{\pi_{+}}{m_{\mathrm{e}}}+(1-\eta) \frac{K_{+}}{m_{\mathrm{H}}}+\frac{\hbar q_{+}}{m_{\mathrm{e}}}, \quad F_{\mathrm{e}-}=\frac{\pi_{-}}{m_{\mathrm{e}}}+(1-\eta) \frac{K_{-}}{m_{\mathrm{H}}} \\
F_{\mathrm{p} \pm}=\frac{\Pi_{ \pm}}{m_{\mathrm{p}}}-(1-\eta) \frac{K_{ \pm}}{m_{\mathrm{H}}}, \\
F_{\mathrm{e} z}=\frac{1}{m_{\mathrm{e}}}\left(p_{z}+\hbar q_{z} / 2\right), \quad F_{\mathrm{p} z}=p_{z} / m_{\mathrm{p}} .
\end{gathered}
$$

In order to proceed with equation (8), we need to calculate the transverse matrix elements $\left\langle n^{\prime} s^{\prime} \eta^{\prime}|\hat{M}| n s \eta\right\rangle_{\perp}$. In the particular case of the dipole approximation $(q \rightarrow 0)$, such calculation has been performed by BP94. In the general case, we use the following result obtained with the same operator technique as in BP94:

$$
\left\langle n^{\prime} s^{\prime} \eta^{\prime}, \boldsymbol{K}_{\perp}+\boldsymbol{q}_{\perp}\left|\exp \left[-\frac{\mathrm{i}}{2} \frac{m_{\mathrm{p}}-m_{\mathrm{e}}}{m_{\mathrm{H}}}\left(\eta^{\prime}-\eta\right) \boldsymbol{K} \boldsymbol{r}_{\perp}+\mathrm{i} \tilde{\boldsymbol{q}} \boldsymbol{r}_{\perp} / 2\right]\right| n s \eta, \boldsymbol{K}_{\perp}\right\rangle_{\perp}=J_{n n^{\prime}}\left(\xi_{\mathrm{e}}\right) J_{n+s, n^{\prime}+s^{\prime}}\left(\xi_{\mathrm{p}}\right)
$$


where $\tilde{\boldsymbol{q}}$ is arbitrary,

$$
\begin{aligned}
& \xi_{\mathrm{e}}=-\mathrm{i} a_{\mathrm{m}}\left(\frac{m_{\mathrm{e}}}{m_{\mathrm{H}}}\left(\eta^{\prime}-\eta\right) K_{+} / \hbar+\left(\tilde{q}_{+}+\eta^{\prime} q_{+}\right) / 2\right), \\
& \xi_{\mathrm{p}}=-\mathrm{i} a_{\mathrm{m}}\left(\frac{m_{\mathrm{p}}}{m_{\mathrm{H}}}\left(\eta^{\prime}-\eta\right) K_{-} / \hbar-\left(\tilde{q}_{-}-\eta^{\prime} q_{-}\right) / 2\right),
\end{aligned}
$$

and

$$
J_{n n^{\prime}}\left(|\xi| \mathrm{e}^{\mathrm{i} \varphi}\right)=\mathrm{e}^{\mathrm{i}\left(n-n^{\prime}\right) \varphi} I_{n n^{\prime}}\left(|\xi|^{2}\right),
$$

$I_{n n^{\prime}}$ being a Laguerre function (Sokolov \& Ternov 1968).

Using equations (A4), (A3a), (A3b), and $(\mathrm{A} 6)$ for $\eta^{\prime}=1$, and neglecting an insignificant common phase, we finally arrive at the expression for the interaction matrix element (8):

$$
\langle f|\hat{M}| i\rangle=2 \sqrt{\gamma} \sum_{n^{\prime} s^{\prime} n s}\left[e_{+} M_{n^{\prime} s^{\prime} n s}^{(-)}+e_{-} M_{n^{\prime} s^{\prime} n s}^{(+)}+e_{z} M_{n^{\prime} s^{\prime} n s}^{(z)}\right]
$$

where

$$
\begin{aligned}
M_{n^{\prime} s^{\prime} n s}^{(-)} & =-\left[\sqrt{n} J_{n-1, n^{\prime}}\left(\xi_{\mathrm{e},+}\right)+\zeta_{\mathrm{e},-} J_{n n^{\prime}}\left(\xi_{\mathrm{e},+}\right)\right] J_{n+s, n^{\prime}+s^{\prime}}\left(\xi_{\mathrm{p},+}\right) \mathcal{Z}_{n^{\prime} s^{\prime} n s}^{(\mathrm{e})} \\
& -\frac{m_{\mathrm{e}}}{m_{\mathrm{p}}} \exp \left(-\mathrm{i} \eta \boldsymbol{r}_{c} \boldsymbol{q}\right)\left[\sqrt{n+s+1} J_{n+s+1, n^{\prime}+s^{\prime}}\left(\xi_{\mathrm{p},-}\right)-\zeta_{\mathrm{p},-} J_{n+s, n^{\prime}+s^{\prime}}\left(\xi_{\mathrm{p},-}\right)\right] \\
& \times J_{n n^{\prime}}\left(\xi_{\mathrm{e},-}\right) \mathcal{Z}_{n^{\prime} s^{\prime} n s}^{(\mathrm{p})}, \\
M_{n^{\prime} s^{\prime} n s}^{(+)} & =\left[\sqrt{n+1} J_{n+1, n^{\prime}}\left(\xi_{\mathrm{e},+}\right)-\zeta_{\mathrm{e},+} J_{n n^{\prime}}\left(\xi_{\mathrm{e},+}\right)\right] J_{n+s, n^{\prime}+s^{\prime}}\left(\xi_{\mathrm{p},+}\right) \mathcal{Z}_{n^{\prime} s^{\prime} n s}^{(\mathrm{e})} \\
& +\frac{m_{\mathrm{e}}}{m_{\mathrm{p}}} \exp \left(-\mathrm{i} \eta \boldsymbol{r}_{c} \boldsymbol{q}\right)\left[\sqrt{n+s} J_{n+s+1, n^{\prime}+s^{\prime}}\left(\xi_{\mathrm{p},-}\right)+\zeta_{\mathrm{p},+} J_{n+s, n^{\prime}+s^{\prime}}\left(\xi_{\mathrm{p},-}\right)\right] \\
& \times J_{n n^{\prime}}\left(\xi_{\mathrm{e},-}\right) \mathcal{Z}_{n^{\prime} s^{\prime} n s}^{\mathrm{p})}, \\
M_{n^{\prime} s^{\prime} n s}^{(z)} & =\left[\widetilde{\mathcal{Z}}_{n^{\prime} s^{\prime} n s}^{(\mathrm{e})}+\frac{\mathrm{i}}{2} a_{\mathrm{m}} q_{z} \mathcal{Z}_{n^{\prime} s^{\prime} n s}^{(\mathrm{e})}\right] J_{n n^{\prime}}\left(\xi_{\mathrm{e},+}\right) J_{n+s, n^{\prime}+s^{\prime}}\left(\xi_{\mathrm{p},+}\right) \\
& +\frac{m_{\mathrm{e}}}{m_{\mathrm{p}}} \exp \left(-\mathrm{i} \eta \boldsymbol{r}_{c} \boldsymbol{q}\right)\left[\widetilde{\mathcal{Z}}_{n^{\prime} s^{\prime} n s}^{(\mathrm{p})}-\frac{\mathrm{i}}{2} a_{\mathrm{m}} q_{z} \mathcal{Z}_{n^{\prime} s^{\prime} n s}^{(\mathrm{p})}\right] J_{n n^{\prime}}\left(\xi_{\mathrm{e},-}\right) J_{n+s, n^{\prime}+s^{\prime}}\left(\xi_{\mathrm{p},-}\right)
\end{aligned}
$$

In these equations

$$
\begin{aligned}
\zeta_{\mathrm{e},+} & =-\frac{\mathrm{i} a_{\mathrm{m}}}{\hbar}\left(\frac{m_{\mathrm{e}}}{m_{\mathrm{H}}}(1-\eta) K_{+}+\hbar q_{+}\right), \quad \zeta_{\mathrm{e},-}=-\frac{\mathrm{i} a_{\mathrm{m}}}{\hbar} \frac{m_{\mathrm{e}}}{m_{\mathrm{H}}}(1-\eta) K_{-}, \\
\zeta_{\mathrm{p}, \pm} & =-\frac{\mathrm{i} a_{\mathrm{m}}}{\hbar} \frac{m_{\mathrm{p}}}{m_{\mathrm{H}}}(1-\eta) K_{ \pm},
\end{aligned}
$$

$\xi_{\mathrm{e}, \pm}$ and $\xi_{\mathrm{p}, \pm}$ are given by equations (A7) with $\eta^{\prime}=1$ and $\tilde{\boldsymbol{q}}= \pm \boldsymbol{q}$, respectively, and the factors

$$
\begin{array}{cl}
\mathcal{Z}_{n^{\prime} s^{\prime} n s}^{(\mathrm{e})}=\left\langle n^{\prime} s^{\prime}, f\left|\mathrm{e}^{\mathrm{i} q_{z} z}\right| n s \eta, i\right\rangle_{\|}, & \mathcal{Z}_{n^{\prime} s^{\prime} n s}^{(\mathrm{p})}=\left\langle n^{\prime} s^{\prime}, f \mid n s \eta, i\right\rangle_{\|}, \\
\widetilde{\mathcal{Z}}_{n^{\prime} s^{\prime} n s}^{(\mathrm{e})}=\left\langle n^{\prime} s^{\prime}, f\left|\mathrm{e}^{\mathrm{i} q_{z} z} a_{\mathrm{m}} \frac{\partial}{\partial z}\right| n s \eta, i\right\rangle_{\|}, & \widetilde{\mathcal{Z}}_{n^{\prime} s^{\prime} n s}^{(\mathrm{p})}=\left\langle n^{\prime} s^{\prime}, f\left|a_{\mathrm{m}} \frac{\partial}{\partial z}\right| n s \eta, i\right\rangle_{\|}
\end{array}
$$

are the longitudinal matrix elements subject to numerical evaluation.

We have presented the result for $\eta^{\prime}=1$. For arbitrary $\eta^{\prime}$, the matrix elements are obtained in analytic form using equation (A15) of BP94 and our equations (A6), (A7) with $\tilde{\boldsymbol{q}}=\left[2 m_{\mathrm{e}}+\eta^{\prime}\left(m_{\mathrm{p}}-m_{\mathrm{e}}\right)\right] \boldsymbol{q} / m_{\mathrm{H}}$ and $\tilde{\boldsymbol{q}}=-\left[2 m_{\mathrm{p}}+\right.$ $\left.\eta^{\prime}\left(m_{\mathrm{e}}-m_{\mathrm{p}}\right)\right] \boldsymbol{q} / m_{\mathrm{H}}$. The result is a straightforward generalization of equations (A10). 


\section{REFERENCES}

Bezchastnov, V. G., \& Potekhin, A. Y. 1994, J. Phys. B, 27, 3349 (BP94)

Bulik, T., \& Pavlov, G. G. 1996, ApJ, 469, 373

Burkova, L. A., Dzyaloshinskii, I. E., Drukarev, G. F., \& Monozon, B. S. 1976, Sov. Phys.-JETP, 44, 276

Däppen, W., Anderson, L. S., \& Mihalas, D. 1987, ApJ, 319, 195

Gnedin, Yu. N., Pavlov, G. G., \& Tsygan, A. I. 1974, Sov. Phys.-JETP, 39, 301

Gorkov, L. P., \& Dzyaloshinskii, I. E. 1968, Sov. Phys.--JETP, 26, 449

Hummer, D. G., \& Mihalas, D. 1988, Ap.J., 331, 794

Johnson, B. R., Hirschfelder, J. O., \& Yang, K.-H. 1983, Rev. Mod. Phys., 55, 109

Kopidakis, N., Ventura, J., \& Herold, H. 1996, A\&A, 308, 747 (KVH96)

Miller, M. C., \& Neuhauser, D. 1991, MNRAS, 253, 107

Ögelman, H. 1995, in The Lives of the Neutron Stars, eds. M. A. Alpar, Ü. Kiziloğlu, \& J. van Paradijs (Dordrecht: Kluwer), 101

Pethick, C. J. 1992, Rev. Mod. Phys., 64, 1133

Pavlov, G. G., \& Mészáros, P. 1993, ApJ, 416, 752 (PM93)

Pavlov, G. G., Shibanov, Yu. A., Zavlin, V. E., \& Meyer, R. D. 1995, in The Lives of the Neutron Stars, eds. M. A. Alpar, Ü. Kiziloğlu, \& J. van Paradijs (Dordrecht: Kluwer), 71

Pavlov, G. G., \& Potekhin, A. Y. 1995, ApJ, 450, 883 (PP95)

Potekhin, A. Y. 1994, J. Phys. B, 27, 1073 (P94)

Potekhin, A. Y., Pavlov, G. G. 1993, ApJ, 407, 330 (PP93)

Potekhin, A. Y., Pavlov, G. G., \& Ventura, J. 1997, A\&A, 317, 618 (PPV97)

Romani, R.W. 1987, ApJ, 313, 718

Schmitt, W., Herold, H., Ruder, H., Wunner, G. 1981, A\&A, 94, 194

Sokolov, A. A., \& Ternov, I. M. 1968, Synchrotron Radiation (Berlin: Academic)

Ventura, J., Herold, H., Ruder, H., \& Geyer, F. 1992, A\&A, 261, 235

Vincke, M., Le Dourneuf, M., \& Baye, D. 1992, J. Phys. B, 25, 2787

Wunner, G., Ruder, H., Herold, H., \& Schmitt, W. 1983, A\&A, 117, 156

This 2-column preprint was prepared with the AAS LATEX macros v4.0. 


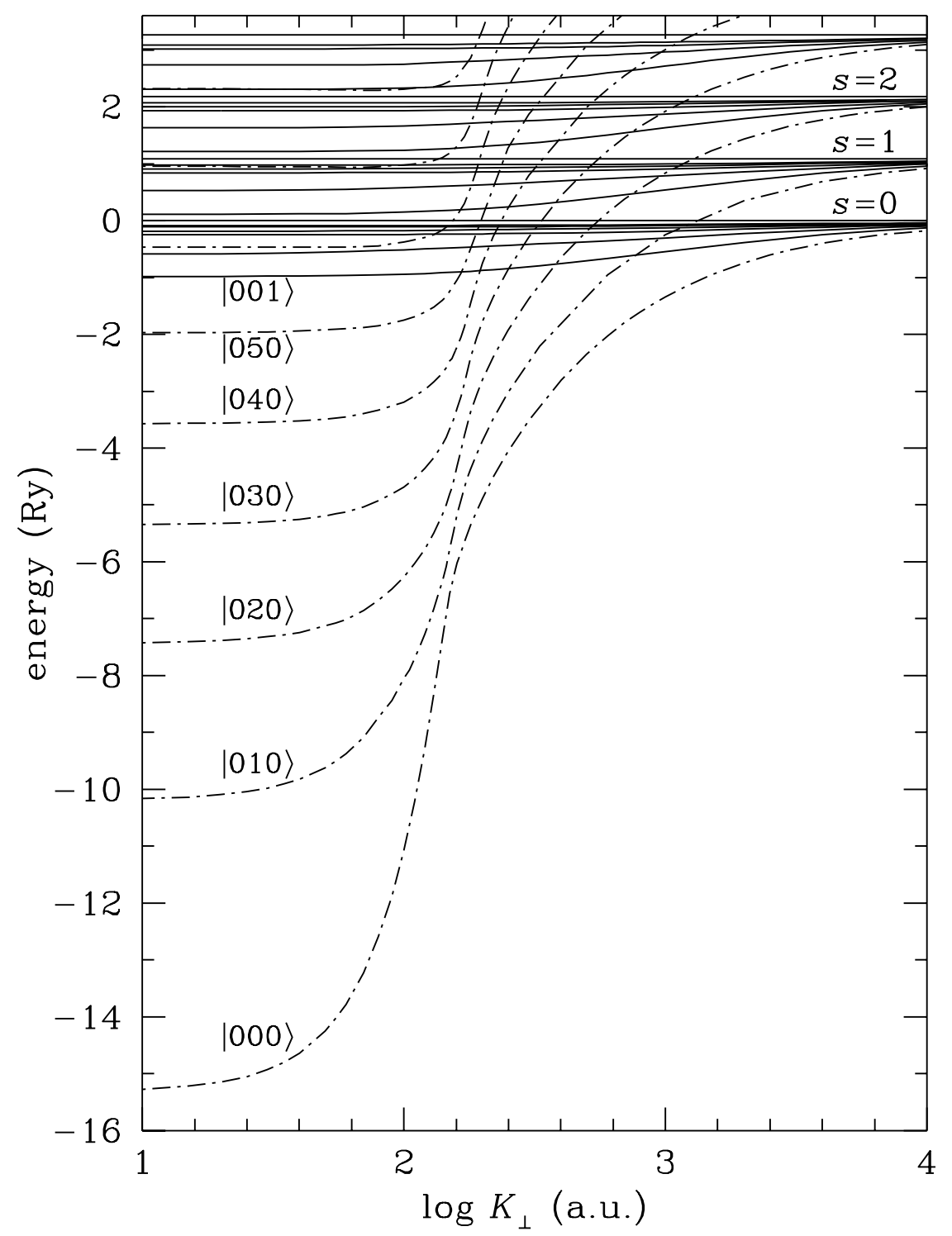

Fig. 1. - Energy spectrum $\left(E_{0 s \nu}-E_{00}^{\perp}\right)$ vs. transverse generalized momentum $K_{\perp}$ at $B=2.35 \times 10^{12} \mathrm{G}$. The curves (dot-dashed for tightly-bound states and solid for hydrogen-like states) are labeled with the quantum numbers, $|0 s \nu\rangle$. 

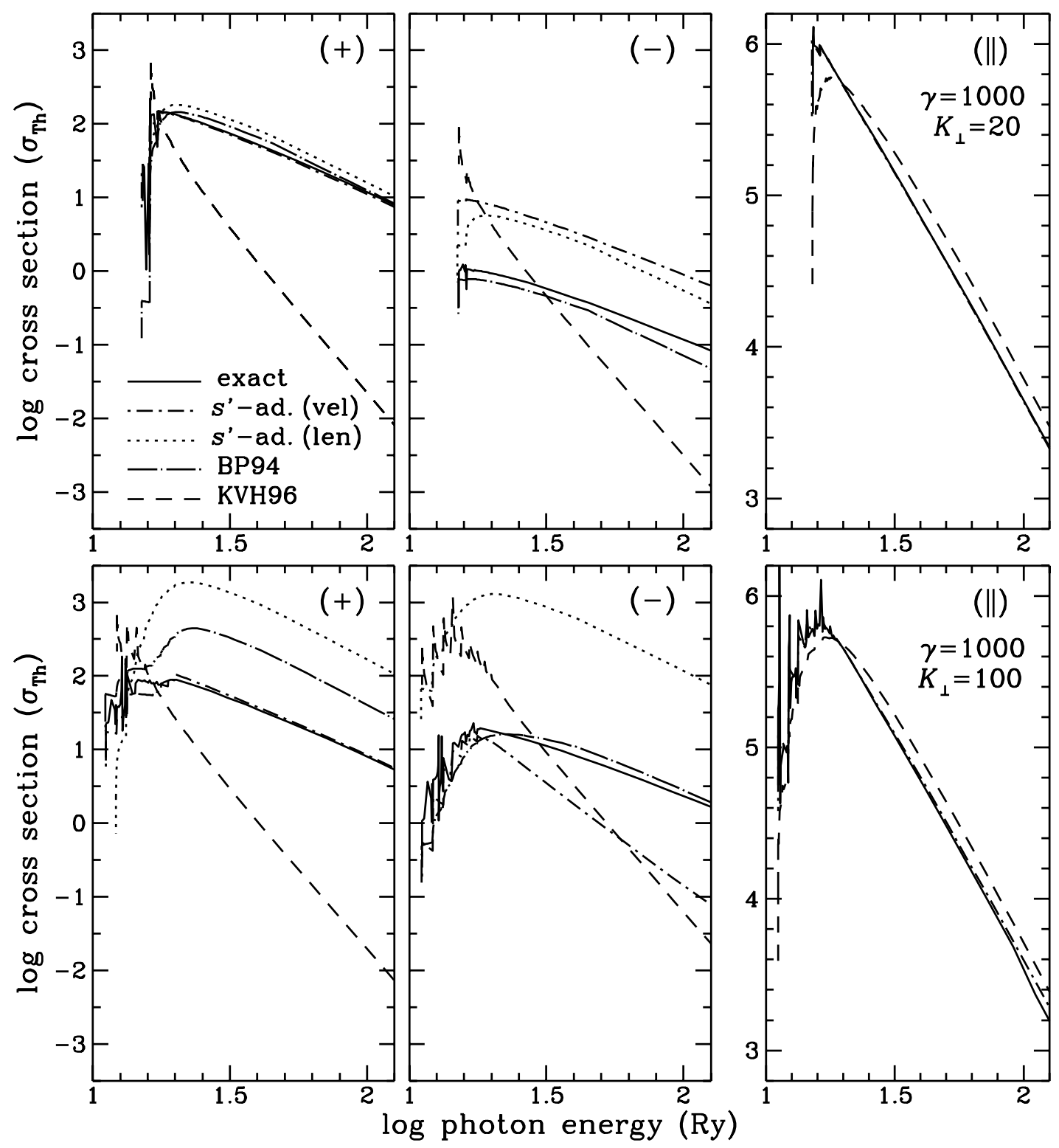

Fig. 2.- Energy dependence of the photoionization cross sections (in units of the Thomson cross section $\sigma_{\mathrm{Th}}$ ) of the ground-state $\mathrm{H}$ atom moving across the magnetic field $B=2.35 \times 10^{12} \mathrm{G}$ with the generalized momentum $K_{\perp}=20$ a.u. $\left(\kappa \equiv K_{\perp} a_{\mathrm{m}} /(\hbar \sqrt{2})=0.447\right.$, upper panels $)$ and $K_{\perp}=100$ a.u. $(\kappa=2.236$, lower panels $)$ for the right $(+)$, left $(-)$, and longitudinal $(\|)$ polarizations (the incident photons move along the magnetic field in the former two cases, and across the field in the latter case). Our numerical results (solid lines) are compared with several common approximations (see text). 

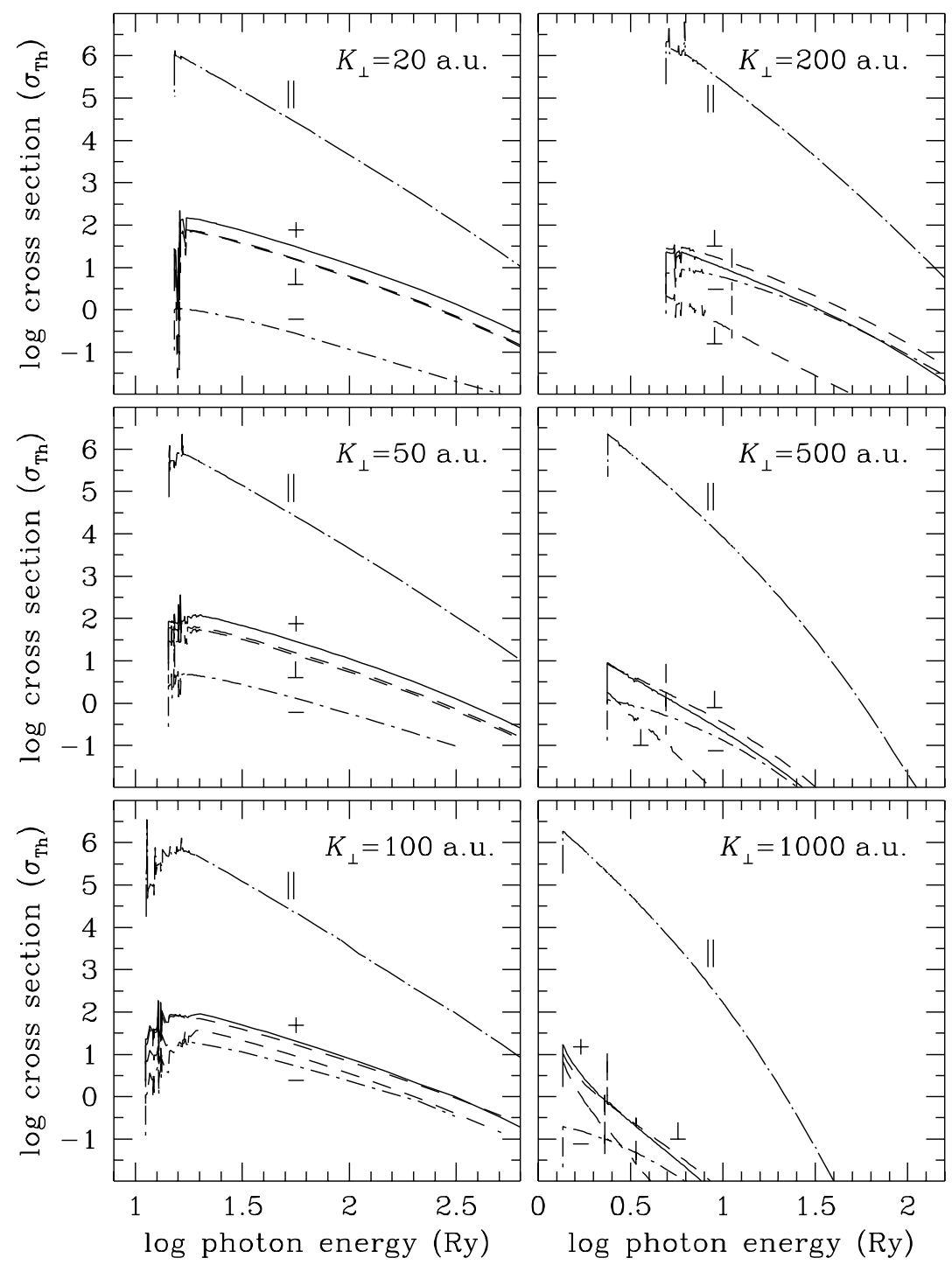

Fig. 3.- Photoionization cross sections of the ground-state $\mathrm{H}$ atoms moving with different $K_{\perp}$ in the magnetic field $B=2.35 \times 10^{12} \mathrm{G}$. Solid and dash-dot lines correspond to the right $(+)$ and left $(-)$ circular polarizations of photons propagating along the magnetic field; long-dash-dot and dashed lines correspond to the linear polarizations parallel $(\|)$ and perpendicular $(\perp)$ to the field, for the transverse propagation. Upper and lower dashed curves are for the wavevector parallel and perpendicular to the transverse component of the generalized momentum. 

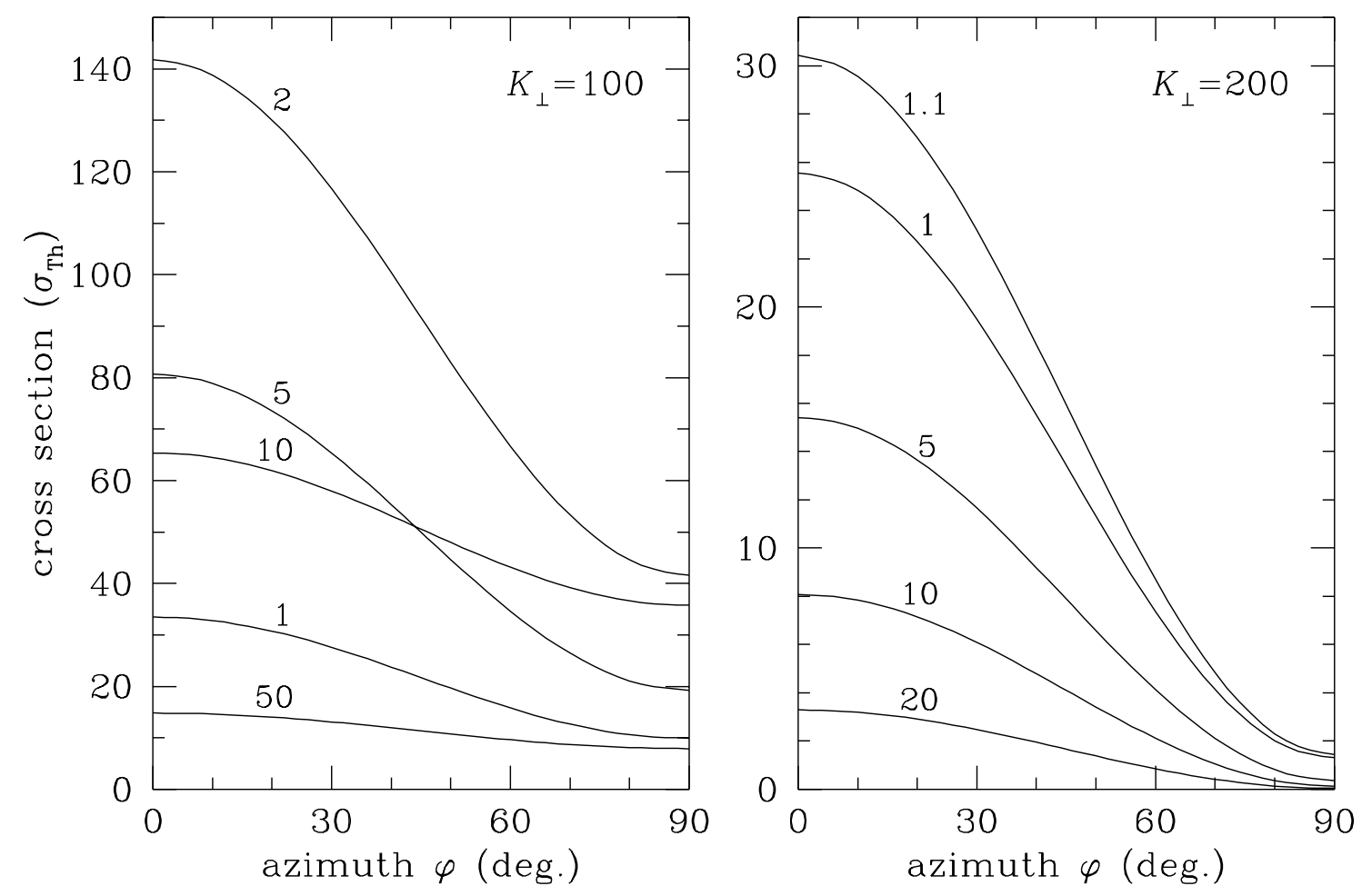

Fig. 4.- Angular dependence of the photoionization cross section $\sigma_{\perp}$ of the ground-state $\mathrm{H}$ atoms moving with $K_{\perp}=100$ and 200 a.u. in the magnetic field $B=2.35 \times 10^{12} \mathrm{G}$. The wavevector and polarization direction are perpendicular to the magnetic field. Numbers near the curves indicate energies $E_{f}$ of the final state (in Ry). 

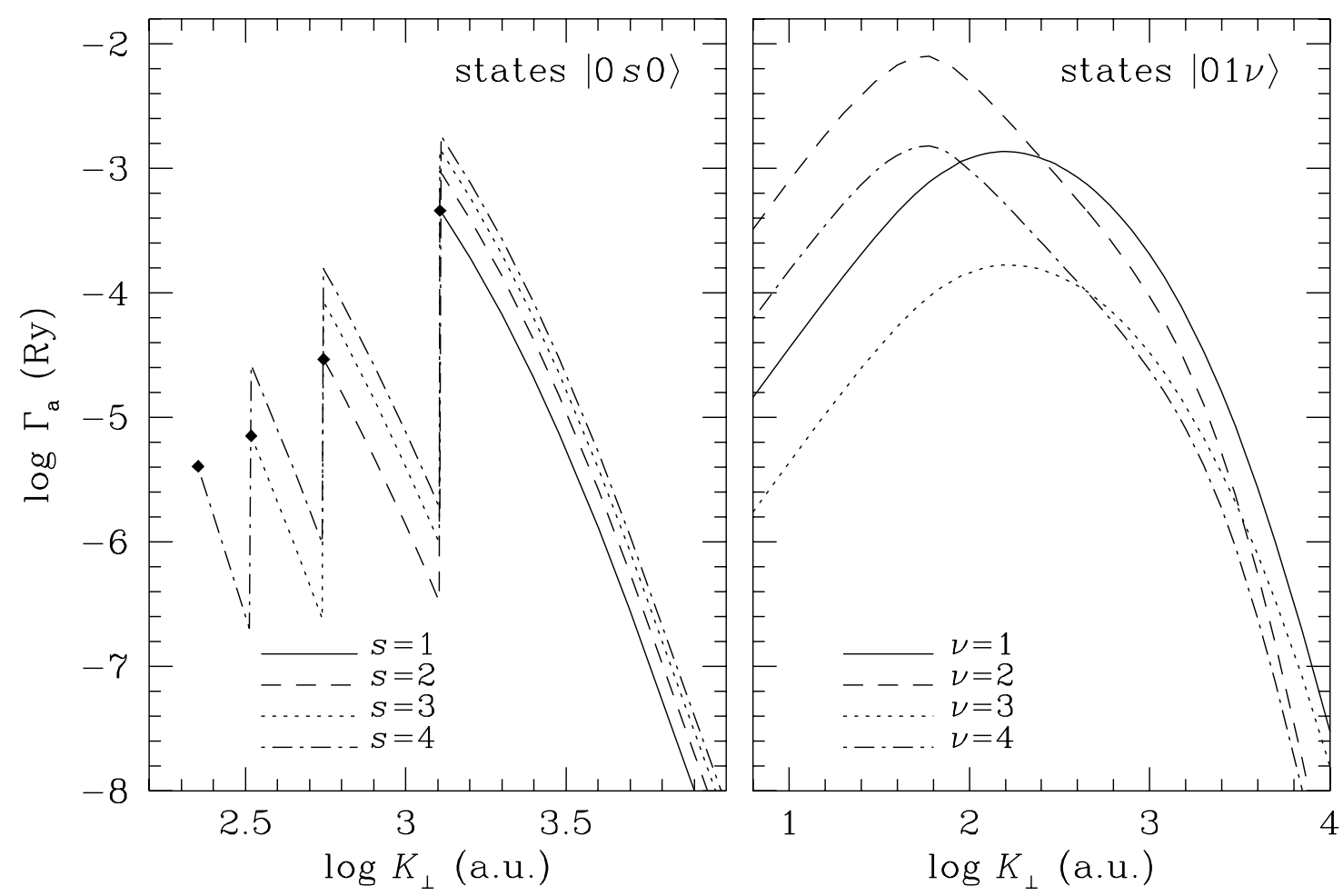

Fig. 5.- Autoionization widths of several tightly-bound (left panel) and hydrogen-like (right panel) atomic resonances as function of $K_{\perp}$ for $B=2.35 \times 10^{12} \mathrm{G}$. Each curve on the left panel starts from the point where the coresponding state enters continuum. 

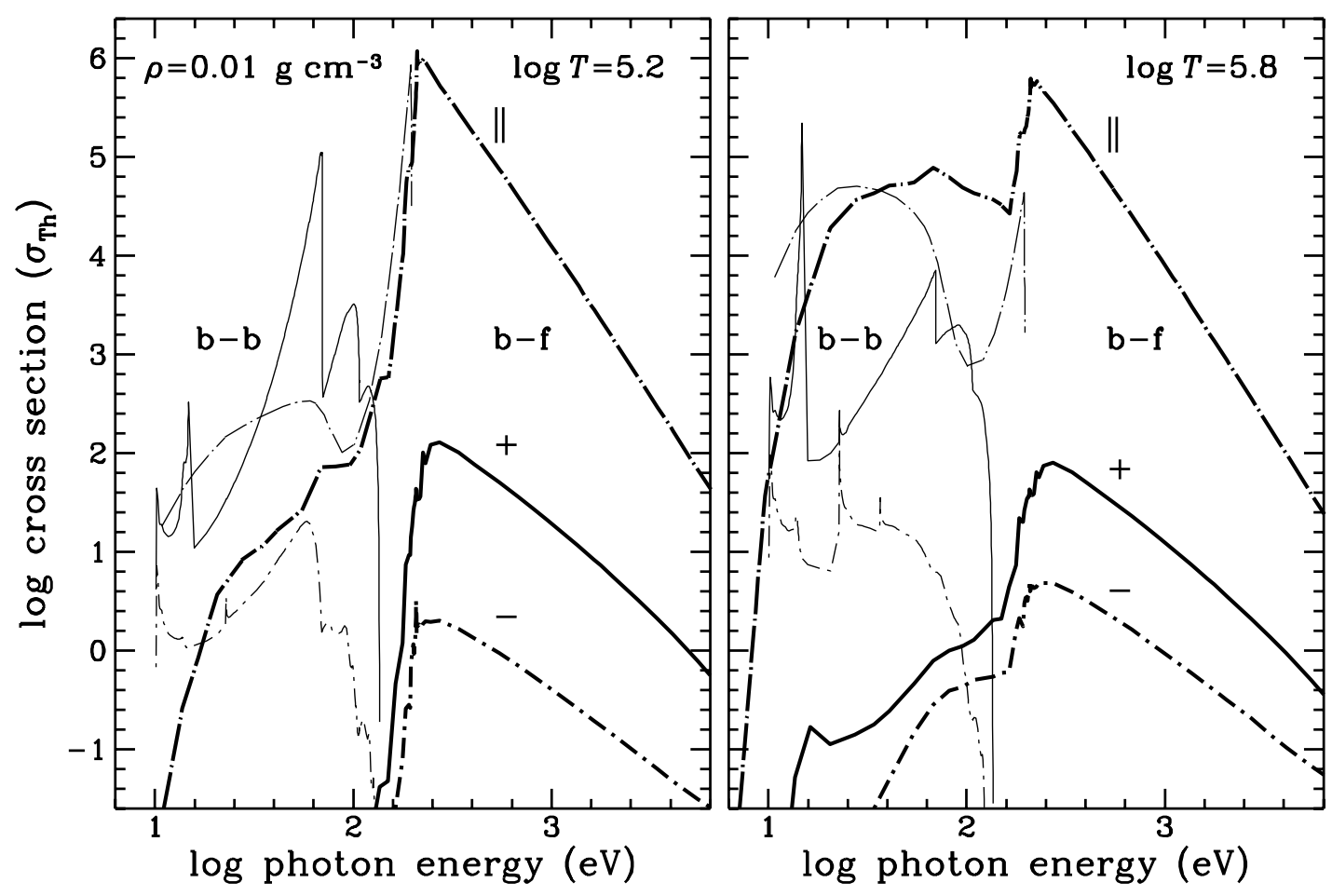

Fig. 6.- Photoionization cross sections (heavy lines) for three polarizations (drawn with the same line styles as in Fig. 3), averaged over the thermal distribution of atoms at the magnetic field $B=2.35 \times 10^{12} \mathrm{G}$, density $\rho=0.01 \mathrm{~g} \mathrm{~cm}^{-3}$, and temperatures $T=10^{5.2} \mathrm{~K}$ and $10^{5.8} \mathrm{~K}$. Light lines represent cross sections arising from the bound-bound transitions (PP95). 


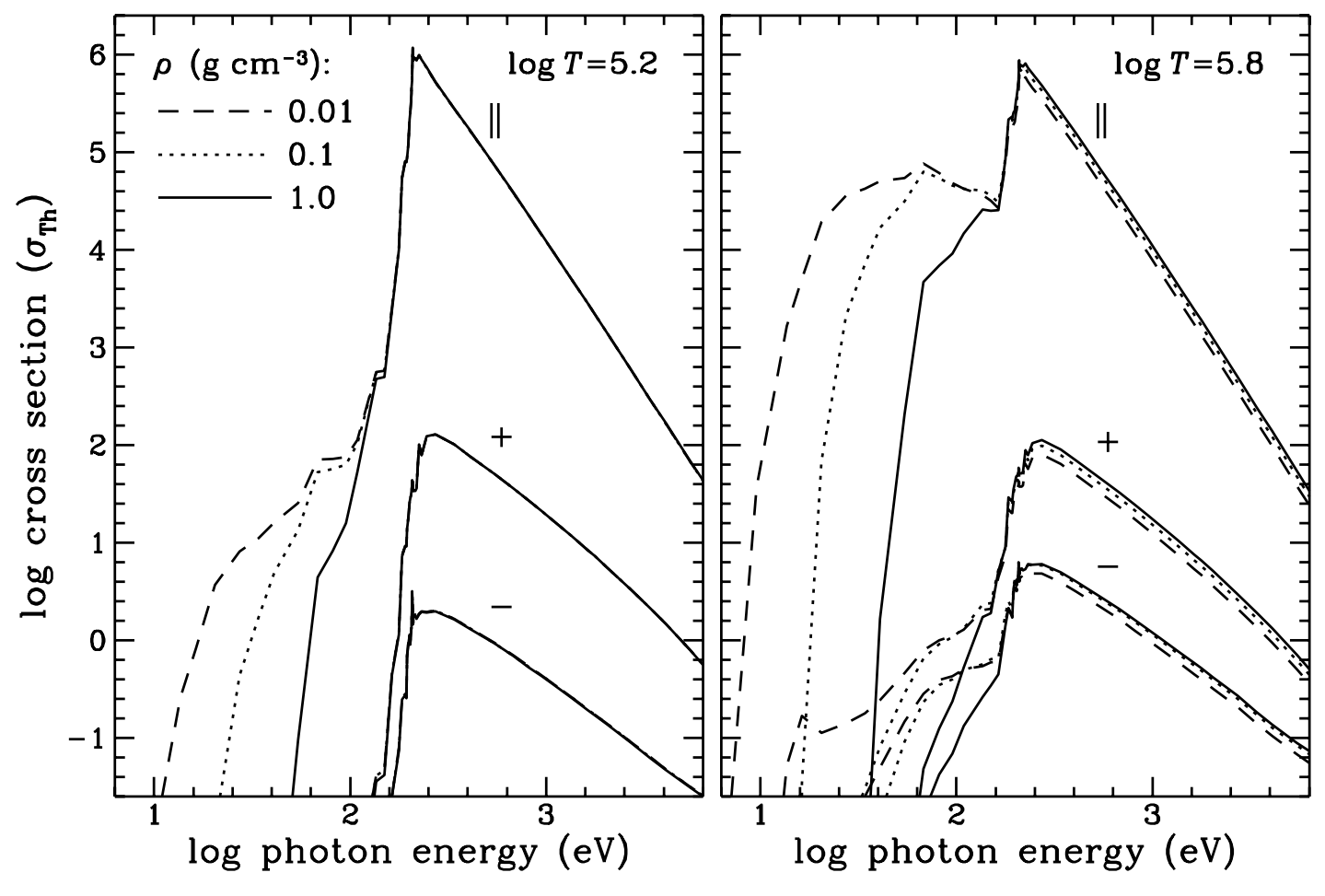

Fig. 7.- Averaged photoionization cross sections for three polarizations, at $B=2.35 \times 10^{12} \mathrm{G}$, three values of $\rho$, and two values of $T$. 

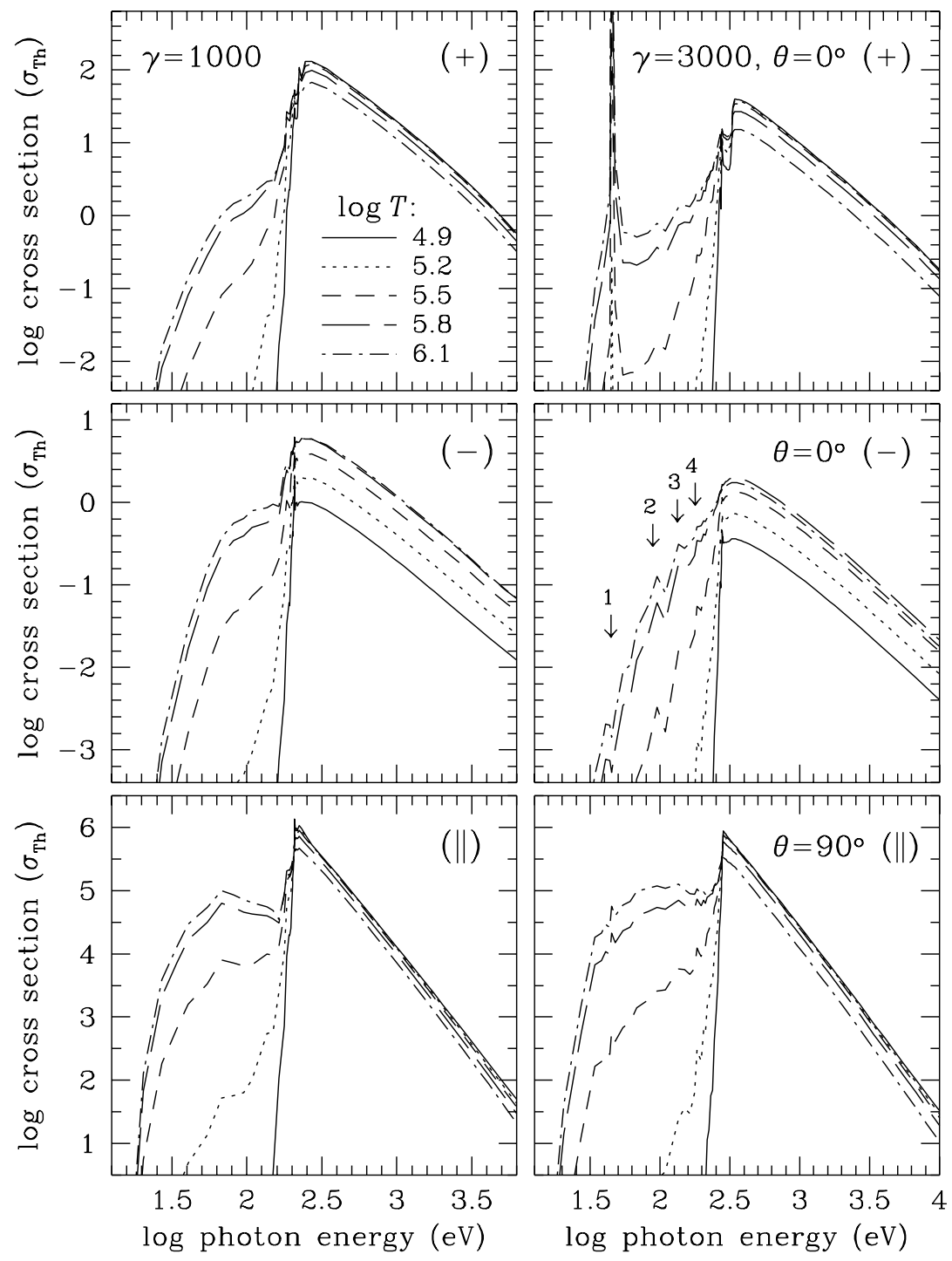

Fig. 8.- Averaged photoionization cross sections for the right (top panels), left (middle panels), and longitudinal (bottom panels) polarizations for $\rho=0.1 \mathrm{~g} \mathrm{~cm}^{-3}, B=2.35 \times 10^{12} \mathrm{G}$ and $7 \times 10^{12} \mathrm{G}$ (left and right panels, respectively), and different temperatures. 